\title{
Novel Meta-Structures for Am Bioengineering Applications
}

\section{Todd C Doehring* and William J Nelson II}

CEO/CSO, Advanced Bioengineering and Meta Systems Labs, USA

Received: 制 February 01, 2018; Published: 潐 February 13, 2018

*Corresponding author: Todd C Doehring, Todd C Doehring, PhD, CEO/CSO, Advanced BioEngineering and Meta Systems LLC, Cleveland, USA, Tel: 215-385-4568; Email: tcd@abemis.com

\begin{abstract}
Additive Manufacturing (AM), also termed 3D-printing, has recently exploded-with rapidly evolving processes, systems, and new bio-compatible materials. At ABĒMIS labs, we have been working on a novel AM enabling meta-structure that, to our knowledge, has not been previously described. Meshagons ${ }^{\mathrm{TM}}$, as we call them, are the first programmable, fractal-like meta-mesh structures that are fully manifold and multi-scale. Description and examples are presented.
\end{abstract}

\section{Introduction}

Additive Manufacturing (AM, 3Dprinting) technologies are recently enabling extraordinary new implant and prosthetic designs. Materials for AM have recently reached medical-grade (e.g. titanium, PEEK, hydroxyapatite) and are progressing [1]. Several companies and research/university organizations have demonstrated exciting AM implant products. A selected few are shown in Figure 1 [2-4]. We note that these, and to our knowledge, all others are latticebased truss structures, generally having some kind of shell with a lattice or truss type infill to reduce weight, and encourage bonein growth. Although improved over a simple surface treated solid, lattices/trusses have topological and material limitations. Lattices, for example, cannot easily conform to complex bio-topological boundaries (complex shapes, holes, etc.) or multi-materials, or related boundary conditions and currently require significant manual intervention/design. To address these limitations, we present here Meshagons ${ }^{\mathrm{T} * *}$, a new type of procedurally-generated finite element (FE) based mesh-meta-structure.

A

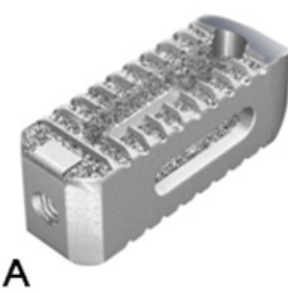

B

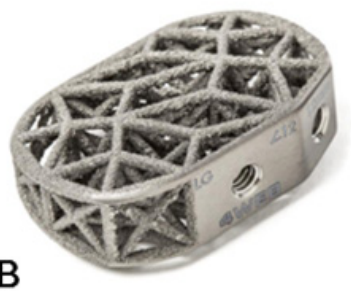

C

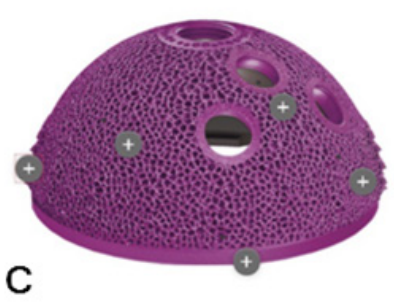

Figure 1: 3D-printed spine and hip implants from (A) Stryker Inc., Tritanium series spine [2] (B) 4WEB Inc., LG series disc replacement [3], (C) NEXTSTEP Arthropedix Inc., Acetabular cup systems [4]. Images are credit to their respective websites (see refs.).

\section{Methods}

A Meshagon $^{\mathrm{TM}}$ is a hyper-mesh. There are three main steps for generating a Meshagon (Figure 2). Briefly:

a) Take any solid model, such as femoral implant (e.g. from CAD, CT/MRI, or any STL file). b) Convert using our FE meshing into a tetrahedral/hex/ hybrid mesh, optimized (proprietary).

c) Transform the FE edge-node mesh into a manifold metamesh structure: Level-1 Meshagon.

We can repeat this process as many times as needed. For 
example, we can take one of the complex joints of the Level-1 Meshagon and re-apply the procedure (Figure 3). We call this a Level-2 Meshagon, and so on... A Meshagon of the size in Figure 1 (appx. 10K elements, 5M triangles) currently takes about $30 \mathrm{~min}$ to process/generate on an Intel i7 with dual Nvidia $1070 \mathrm{GPU}$ processors. Improvements in computational speed are ongoingt.

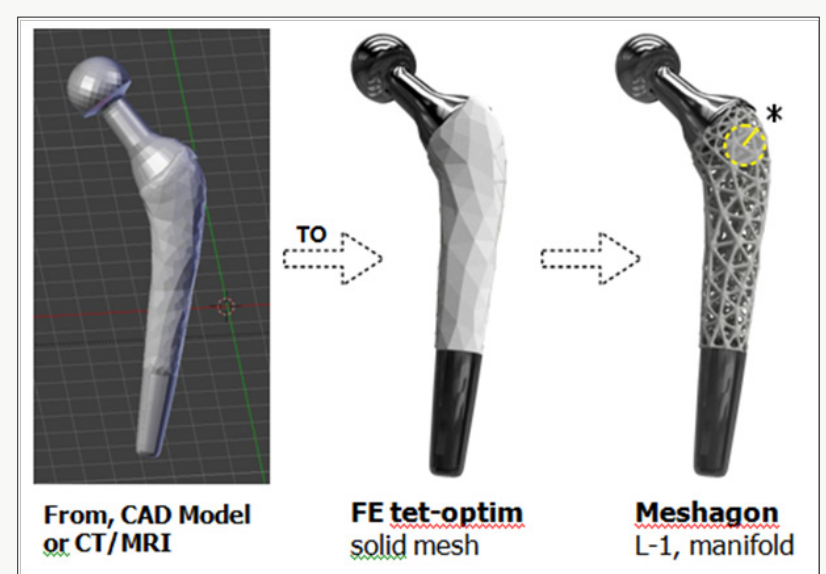

Figure 2: Meshagon generation; 3 main steps (from left). Start with a CAD or CT/MRI model of astructure such as this prototype femoral implant $==>$ Generate FE solid tetrahedral or hybrid mesh, optimized for structure/loads/BCs and Meshagon generation ==> L-1 Meshagon is fully manifold $\dagger$.
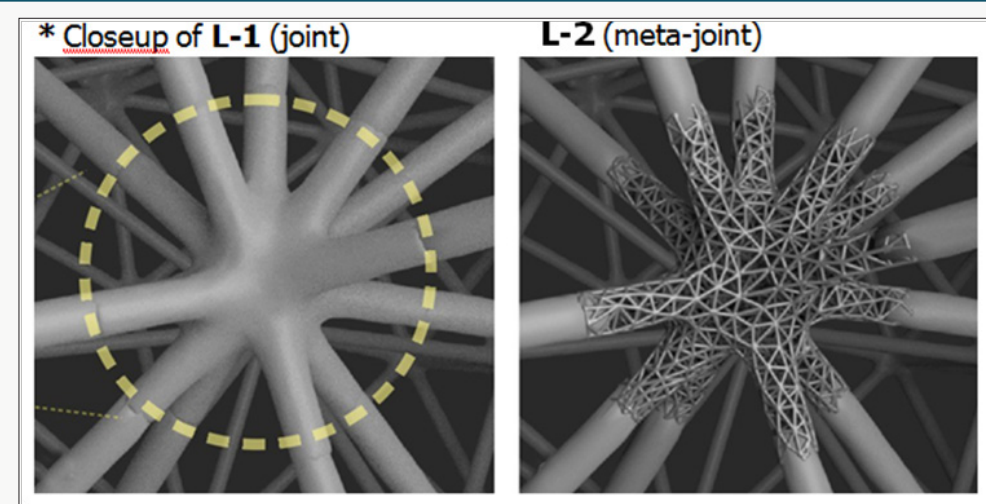

Figure 3: Close-up of internal joint (left, as indicated in Fig. 2) and L-2 Meshagon (right). Parameters for each Level can be independent and/or dependent, depending on the desired simple or complex functional model(s). For example, L-1 can be made more dense and L-2 less dense, etc. Any parameter can be added to the topology and the chosen underlying model(s)t.

\section{Results/Examples}

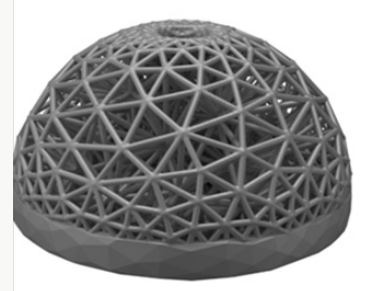

A. Meshagon L-1 acetabulum (socket) implant prototype.

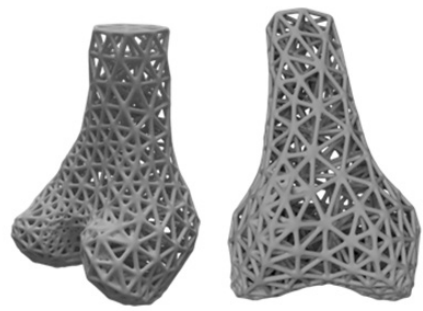

B. L-1 Distal femur scaffold.* Geometry from CT-scan.

Figure 4: Two examples of Meshagon implant prototypes: (left) An acetabular cup Meshagon, note that this is just one densityall parameters can be changed and Meshagon updated accordingly. (right) Two views of a prototype distal knee scaffold; (credit: CT and segmentation from the SimTK Open Knee project [5]). This demonstrates capability for complex structured Meshagon scaffold generation (not as an implant, to be precise). This Meshagon meta-structure could be printed in combination of titanium and hydroxyapatite or other bio-compatible AM materials and related models [6-7]. 
The hip femoral component Level-1 and Level-2 prototypes (Figures 2 \& 3) have good strength characteristics and ultralight weight $(54+\%$ reduced mass, assuming fused titanium or stainless steel material). Strut thickness was held here to $2.5 \mathrm{~mm}$ but of course can be resized, changed, or adapted to suit physical 3D-printer capabilities. The hydroxyapatite coating was simulated and not part of the FE optimization. The acetabular component prototype example (Figure 4a) was designed to be adjustable to topologies/factors important for bone in growth and mesh density for bone-implant integration [5-7]. There is only one central hole in this model (for fixation) but this can be easily modified, holes added, to suit. This early prototype supports $30 x+B W$ in simulation. The distal femur and chondyle model (Figure $4 \mathrm{~b}$ ) is manifold and fully printable, successfully demonstrating unique potential for Meshagon scaffolds that can be parametrically (programmed) as well as conforming to complex topologies.

\section{Discussion}

Meshagons and the related concepts presented here, along with rapidly advancing $\mathrm{AM} / 3 \mathrm{D}$ printing technologies, have promise for generating unprecedented, multi-scale, ultra-light meta-structures. We have produced and printed a number of medical and implant related Meshagon structures as shown.

A significant advantage of Meshagons is the ability to automatically (procedurally) compute multi- scale Meshagons that conform to complex biological shapes/topologies that until now were not possible to achieve (to our knowledge).

\section{Limitations}

We have not performed precision experimental testing on the printed implant structures (e.g. tension-compression, fatigue). The results and discussions here are based upon FE model simulations, and basic tests of actual printed structures. We have done simple compression tests of standard 'brick' Meshagons with positive confirmation of simulation results, but further work and collaboration is needed in this area. Another aspect that needs improvement is defining interpolation between L-levels. We are currently using a simple modified Boolean method, but is now working on improved fully implicit method(s) that will enable better control of (any) combining parameters.

Our recent work enables large scale meta-mesh parallelcalculations, now limited only by computer RAM and SSD storage (multi-scene). We can now generate Meshagons with appx 1M elements (est. 50M triangles) in appx 80 minutes for Level-1*. This can improve (work needed). Also, we can now control the thicknesses of individual struts and joints, the chamfer (or transitions), local and global density-porosity, optimize local stresses and vibrations using modal and explicit analyses. An interesting parallel between Meshagons and actual connective tissues is that they are both heirarchical structures. Tissues have multi-scale topologies, for example, Tendon $\rightarrow$ Fascicle $\rightarrow$ Fiber bundle $\rightarrow$ Fibril $\rightarrow$ Tropocollagen. Meshagon meta-structures are also multi-scale, L1 $\rightarrow$ L2 $\rightarrow$ L3 $\rightarrow$ etc. Thus, Meshagons have a unique potential to improve description of actual multi-scale tissue geometries and related topologies/structure. Another concept we introduce here is the capability of Meshagon "multilevel porosity". The different levels L-1 and L-2 (Figure 3) each have different, porosities and other properties that can be programmed/ controlled. That means with Meshagons we can generate multiporosity structures that may better encourage bone-in growth and/or restorability properties, possibly leading to improved tissue engineered constructs and regeneration. Development is also ongoing for multi-scale, multi-material structures with enhanced optimized physical, electrical, and biochemical properties. New AM materials are now capable of directing and controlling electrical charge, which will open whole new frontiers for active 'smart' Meshagons and related structures. We are also extending to multimesh combinations, and further innovations in AM hyper metamaterials for medical implants and other areas-such as Aerospace, where Meshagon ultra- light, ultra-strength capabilities are also critical factors. Ultimately we believe that AM and Meshagon-type structures (and even the simpler lattice-type), will lead to dramatic improvements in medical implant, prosthetic, and system/device designs in the near future.

\section{Acknowledgment}

Thank you to Prof. Alan Freed (TAMU) and colleagues for exciting discussions and mathematical support. Much thanks to the open source projects: Blender3D.org, OpenCV, EMGU. com, OpenKnee SimTK.org, CGAL.org, Netgen.org, TopMod (Prof. Akleman, TAMU), and all their related assistance. Also thank you to S. Chokhandre (Principal Research Engineer, Cleveland Clinic Foundation) for invaluable critiques and review.

\section{Footnotes}

*Meshagons are trademarked 2017, process patent-pending 2017-18. All code, images and Meshagon printed structures are Copyright 2014-2018, ABĒMIS LLC.

†The procedures described here present the basic Meshagon concept. Certain steps in the FE meshing and Meshagon generation procedure are trade-secret and patent-pending 2017-18. For further information please contact the Authors.

\section{References}

1. Cui X, Breitenkamp K, Finn MG, Lotz M, D'Lima DD (2012) Direct Human Cartilage Repair Using Three-Dimensional Bioprinting Technology. Tissue Engineering Part A 18(11-12): 1304-1312.

2. Stryker Inc, Tritanium series of lumbar fusion systems.

3. 4WEB Medical Inc., series of truss systems.

4. NEXTSTEP Arthropedix Inc. Acetabular cup systems.

5. SimTK, Erdemir A. (2016) Open Knee: open source modeling and simulation in knee biomechanics. J Knee Surg 29: 107-116. 
6. Liebschner M, Bucklen B, Wettergreen M (2005) Mechanical Aspects of Tissue Engineering. Seminars in Plastic Surgery 19(3): 217-228.
7. Freed AD, Einstein DR, Sacks MS (2010) Hypoelastic Soft Tissues: Part II: In-Plane Biaxial Experiments. Acta mechanica 213(1-2): 205-222. (c) (i) This work is licensed under Creative To Submit Your Article Click Here: Submit Article DOI: 10.32474/OAJBEB.2018.01.000107

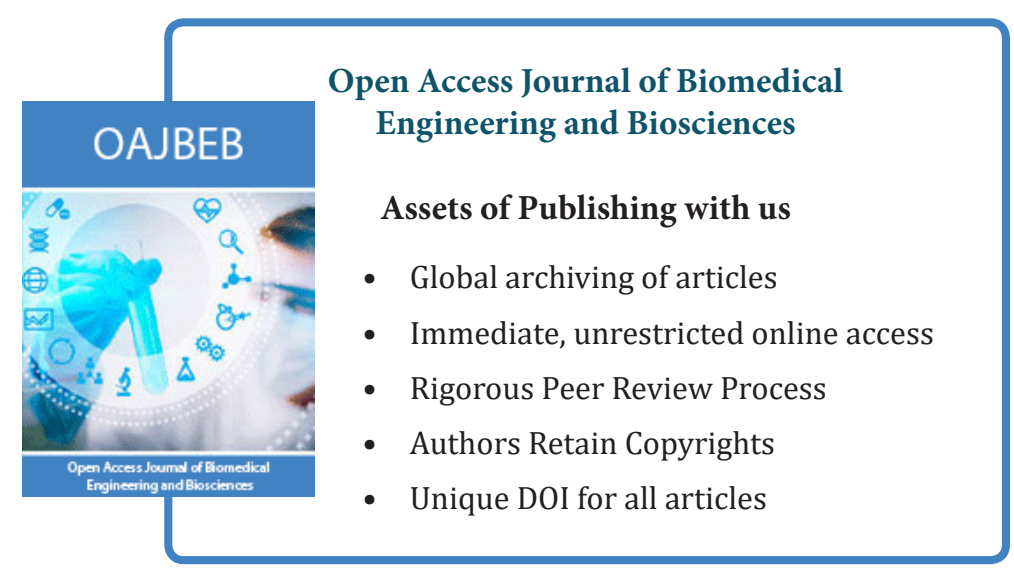

